\title{
Décisions de la Commission d'interprétation paritaire
}

TARMED Suisse

Numéro 07002 Prise en charge
périopératoire et prestations
additionnelles

Position tarif: 00.0010, 00.0050, 00.0110, $00.0140,28.0010,28.0030,28.0040,28.0050$, 28.0060

Valable dès: 19 juin 2007

\section{Interprétation}

L'étude de dossier et l'administration/préparation pour l'opération (annonce pour la narcose, évaluation des formulaires relatifs à la préparation de la narcose, prescriptions de laboratoire et de radiologie, examens spécifiques pour patients à risques et patients dans un contexte difficile, etc.) font partie intégrante de la prestation d'anesthésie et de la prise en charge périopératoire. Le médecin spécialiste en anesthésie ne peut pas facturer la position tarifaire 00.0140 «Prestation médicale en l'absence du patient (y compris étude de dossier)» en sus de ces prestations.

\section{Numéro 0701415.0710 \\ (Etude du sommeil, oxymétrie nocturne ambulatoire informatisée, comme prestation exclusive) \\ Position tarif: 15.0710 \\ Valable dès: 19 juin 2007}

\section{Interprétation}

Etant donné que la CFP n'a jamais statué sur la position tarifaire 15.0710 , la règle imposée par la loi n'est pas susceptible de modifications. Elle s'applique telle quelle selon le texte de la LAA «aucune prestation obligatoire ou prestation obligatoire restreinte».

\section{Numéro 07016 28.0020:}

la majoration ne s'applique qu'à la PM

Position tarif: 28.0020

Valable dès: 19 juin 2007

\section{Interprétation}

Correction de l'interprétation: La majoration de 10\% ne s'applique qu'à la PM.

\author{
Numéro 07017 Cumulation \\ avec la consultation radiologique \\ de base $\mathbf{3 0 . 0 0 1 0}$ \\ Position tarif: 30.0010 \\ Valable dès: 19 juin 2007
}

\section{Interprétation}

Les radiologues des hôpitaux et des instituts peuvent facturer la consultation de base 30.0010. Elle est cumulable avec GP-46, GP-24 et les prestations de base techniques des chapitres 30.02.10, 30.03.08, 30.04.06 et 30.05.05.

\section{Numéro 07018}

Psychothérapie selon OPAS

Position tarif: 02.0030, 02.0050

Valable dès: 19 juin 2007

\section{Interprétation}

En ce qui concerne les positions 02.0030 et 02.0050 , la règle de la quantité de l'interprétation «Pour la psychothérapie médicale, quantité selon l'OPAS, art. 3: au maximum 12 fois par séance et par patient» est obsolète et ne doit plus être appliquée.

\section{Numéro 07019 IC-02.04-1 \\ Indemnisation des prestations psychia- triques ambulatoires et non médicales \\ Position tarif: IC-02.04-1 \\ Valable dès: 19 juin 2007}

\section{Interprétation}

Dans des situations d'urgence ou de crise, la limitation à quatre heures par semaine peut servir de base pour déterminer la valeur moyenne pour trois mois. Les prestations facturées ne doivent pas dépasser la moyenne de quatre heures par semaine réparties en l'espace de trois mois. 